\title{
Studies on Antibiotic Sensitivity Pattern of Pseudomonas aeruginosa Isolated from Hospitalized Patients
}

\author{
Shahidul A. Chowdhury ${ }^{*}$, Jabun Naher' ${ }^{1}$ Abdullah A. Mamun², Ruhul A. Khan ${ }^{2}$, \\ Jannatul Ferdous ${ }^{1}$, Sharmin Sultana ${ }^{1}$ \\ ${ }^{1}$ Department of Microbiology, University of Chittagong, Chittagong, Bangladesh \\ ${ }^{2}$ Radioactivity Testing and Monitoring Laboratory, Bangladesh Atomic Energy Commission, Chittagong, \\ Bangladesh \\ Email: *nawajamin@gmail.com
}

Received 1 July 2014; revised 11 August 2014; accepted 19 September 2014

Copyright (C) 2014 by authors and OALib.

This work is licensed under the Creative Commons Attribution International License (CC BY).

http://creativecommons.org/licenses/by/4.0/

(c) (i) Open Access

\section{Abstract}

This study was performed to evaluate the antibiotic sensitivity pattern and determination of MIC (Minimum inhibitory concentration) and MBC (Minimum bactericidal concentration) value of Pseudomonas aeruginosa. The samples were collected from hospitalized and outdoor patients of Chittagong Maa-O-Shisu General hospital (October, 2010 to April, 2011). From the sample (blood, pus and urine) the isolate was identified on the basis of their morphological, cultural and biochemical characteristics. Antibiotic sensitivity pattern of the isolates was then observed (Disc diffusion method). It was found that Ciprofloxacin, Azithromycin and Chloramphenicol showed the highest percentages of sensitivity (above $60 \%$ ) so they were the most effective drug for the treatment of Pseudomonas aeruginosa infection, where Vancomycin, Amphicillin and Amoxicillin showed the lowest percentages of sensitivity as well as less effective drug. Minimum Inhibitory Concentration (MIC) (Turbidimetric Method) values were determined against six antibiotics (Ciprofloxacin, Azithromycin, Kanamycin, Erythromycin, Chloramphenicol and Gentamycin). The MIC of Ciprofloxacin and Azithromycin for the majority of clinical isolates was 2 to $>64 \mu \mathrm{g} / \mathrm{ml}$. Like that the MIC of Gentamycin, Kanamycin and Chloramphenicol for the clinical isolates was 4 to $>64 \mu \mathrm{g} / \mathrm{ml}$. MIC value of Erythromycin was between 8 to $>64 \mu \mathrm{g}$. The Minimum bactericidal concentration (MBC) for all these six drugs were also done and the result was between 2 to $>64 \mu \mathrm{g} / \mathrm{ml}$.

\section{Keywords}

Antibiotic, Sensitivity, MIC (Minimum Inhibitory Concentration), MBC (Minimum Bactericidal Concentration), Pseudomonas aeruginosa

${ }^{*}$ Corresponding author.

How to cite this paper: Chowdhury, S.A., Naher, J., Mamun, A.A., Khan, R.A., Ferdous, J. and Sultana, S. (2014) Studies on Antibiotic Sensitivity Pattern of Pseudomonas aeruginosa Isolated from Hospitalized Patients. Open Access Library Journal, 1: e911. http://dx.doi.org/10.4236/oalib.1100911 


\section{Introduction}

Pseudomonas aeruginosa is a Gram-negative, mesophilic, aerobic, rod shaped bacteria (length 1.5 - $3.0 \mu \mathrm{m}$ and width 0.5 to $0.8 \mu \mathrm{m}$ ) [1]. It is an opportunistic pathogen and a major cause of hospital acquired nosocomial infections of humans but rarely causes infections in healthy individuals. Even though the bacterium almost never infects uncompromised tissues but if the tissue defenses are compromised in some manner, there is hardly any tissue that it cannot infect [2] [3]. It tends to infect people with immunodeficiency or burns and those with indwelling catheters or respirators. Infection with $P$. aeruginosa can lead to urinary tract infections, sepsis (blood stream infection), pneumonia, endocarditis, pharyngitis, meningitis, and many other medical problems. It colonises the lungs of patients with cystic fibrosis (CF) and contributes to the chronic progressive pulmonary disease and death rate in CF. Although the initial isolation of $P$. aeruginosa from sputum may be intermittent in CF and bronchiectasis, once a chronic infection is established it is almost impossible to eradicate it even with intensive antibiotic treatment [4]. Pseudomonas aeruginosa is notorious for its resistance to antibiotics and is therefore, a particularly dangerous and dreaded pathogen [5]. It has a natural tendency for the development of resistance to antibiotics. This limits future therapeutic uses of antibiotics against this bacterium and increases rates of mortality. The bacterium is naturally resistant to many antibiotics including Tetracycline and Benzylpenicillin due to the permeability barrier afforded by its outer membrane lipopolysaccharide (LPS) [6]. It can colonize surfaces in a biofilm form making the cells impervious to antibiotics. Pseudomonas aeruginosa has been living in the soil for millions of years in the presence of antibiotic producing bacilli, actinomycetes and moulds. Therefore, it has developed resistance to a variety of their naturally occurring antibiotics. Moreover, $P$. aeruginosa maintains antibiotic resistant plasmids, and is able to transfer these genes by means of lateral gene transfer (transduction and conjugation). Only a few antibiotics are effective against Pseudomonas including some $\beta$-lactams, aminoglycosides and fluoroquinolones but these antibiotics are not effective against all strains. The futility of treating Pseudomonas infections with antibiotics is most dramatically illustrated in cystic fibrosis patients, virtually all of whom eventually become infected with a strain that is multi drug resistant and cannot be treated [3]. Pseudomonas aeruginosa produces a variety of virulence factors including protease enzymes, mucoidexopolysaccharide, pili, exotoxin A, lipopolysaccharide, pigments, lipase, haemolysin, histamine, exoenzyme S, leukocidin and rhamnolipids [7]. These aid the bacteria to adhere, invade and colonize to their host by damaging the host's immune responses and forming a barrier to antibiotics. No single virulence factor by itself is potent but the whole array of factors contributes to the pathogenicity of the P. aeruginosa [8].

The objective of the present research was to evaluate the sensitivity pattern and determination of MIC (Minimum inhibitory concentration) and MBC (Minimum bactericidal concentration) value of Pseudomonas aeruginosa.

\section{Materials and Methods}

\subsection{Materials}

The present research work was conducted at Chittagong Maa-O-Shishu General Hospital (CMOSH) during October, 2010 to April, 2011. The isolated Pseudomonas aeruginosa from the samples brought to its Pathology Laboratory and sensitivity pattern was determined against commonly used antibiotics by disc diffusion method [9]. The hospital's laboratory samples comprised of blood, pus and swabs from different body sites, from outdoor patients (OPD) as well as indoor patients (IDP) from different wards of the hospital.

\subsection{Sample Collection and Processing}

Blood, pus and urine samples were collected from patients visiting OPD (Out Patients Department) and different wards (IDP) and were brought to the Pathology laboratory. Freshly drawn, 3 - $5 \mathrm{ml}$ blood sample was immediately transferred to $50 \mathrm{ml}$ of Tryptone Soya broth (CM0129-OXOID) and incubated at $37^{\circ} \mathrm{C}$ for $24 \mathrm{~h}$. In case of no growth, incubation period was extended for another $24 \mathrm{~h}$. In case of growth positive, it was sub-cultured on Ce- 
trimider Agar (CM 0055-OXOID) and MacConkey Agar (CM 0007-OXOID) plates incubated for $24 \mathrm{~h}$ at $37^{\circ} \mathrm{C}$. Pus samples were directly inoculated on Cetrimide Agar (CM 0055-OXOID) and MacConkey Agar (CM 0007OXOID) plates incubated for $24 \mathrm{~h}$ at $37^{\circ} \mathrm{C}$. Urine samples were cultured on Blood agar, MacConkey agar and Cetrimide agar incubated for $24-48 \mathrm{~h}$ at $37^{\circ} \mathrm{C}$. Randomly selected isolates were subcultured and preserved for further studies. For this purpose mid exponential phase bacterial isolates were inoculated into nutrient agar slunt and maintained at $4^{\circ} \mathrm{C}$. In this condition, bacterial culture can be preserved for as long as one month. Isolated colonies, after purification, were initially Gram-stained. The isolates were biochemically characterized and identified up to species level according to Bergey’s Manual of determinative Bacteriology [1].

\subsection{Antibiotic Susceptibility Test (Disc Diffusion)}

The disc diffusion test for Pseudomonas aeruginosa was carried on Mueller-Hinton Agar (CM0337-OXOID) as growth medium. Medium was prepared according to manufacturer's instructions and sterilized by autoclaving at $121^{\circ} \mathrm{C}$ for $15 \mathrm{mins}$. Sterilized medium was then cooled in a water bath. About $25 \mathrm{ml}$ of medium was poured into 90 mm diameter sterile Petri-plates to a depth of $4 \mathrm{~mm}$ on a level surface to make the depth of the medium uniform and left at room temperature overnight to check sterility. Inoculum was spreaded evenly over the entire surface of the Mueller-Hinton Agar plates by swabbing back and forth across the agar in three directions to give a uniform inoculum to the entire surface. These plates were allowed to dry before applying discs. The discs of given potency were applied on the inoculated plates with the help of sterile forceps (Table 1). Then the plates were placed in an incubator at $37^{\circ} \mathrm{C}$ for 18 hours in inverted position. After 18 hours of incubation, plates were examined and the diameters of zone of inhibition were measured in $\mathrm{mm}$ [9].

\subsection{Determination of MIC (Turbidimetric Method)}

Individual antibiotics were dissolved in nutrient broth and diluted (nutrient broth). The MIC range varied with different drugs. All MIC range was followed according to the NCCLS guidelines [10]. Inoculums were obtained from an overnight agar culture of the test organism. Inoculums for the MIC test were prepared by taking at least three to five well-isolated colonies of the same morphology from an agar plate culture. The top of each colony was touched with a sterile loop and the growth was transferred into a tube containing 4 to $5 \mathrm{ml}$ of normal saline. The broth culture was incubated at $35^{\circ} \mathrm{C}$ until it achieved the turbidity of the 0.5 McFarland standards (usually 2 to 6 hours). This results in a suspension containing approximately 1 to $2 \times 10^{8} \mathrm{cfu} / \mathrm{ml}$. The turbidity of the actively growing broth culture was adjusted with the broth to obtain a turbidity comparable to that of 0.5 McFarland standard.

A 0.5 McFarland standard was prepared as described in NCCLS [11]. One percent V/V solution of sulfuric acid

Table 1. Antibiotic disc with given potency that were used in the study.

\begin{tabular}{|c|c|c|c|c|c|}
\hline SL No. & Antimicrobial agent & Antimicrobial group & Code & Disc potency & Manufacturer \\
\hline 01 & Ampicillin & Penicillin & A & $25 \mu \mathrm{g}$ & Hi Media \\
\hline 02 & Amoxicillin & Penicillin & AML & $30 \mu \mathrm{g}$ & Hi Media \\
\hline 03 & Tetracycline & Acetogenin & $\mathrm{T}$ & $30 \mu \mathrm{g}$ & Hi Media \\
\hline 04 & Ciprofloxacin & Quinolones & CIP & $5 \mu \mathrm{g}$ & Hi Media \\
\hline 05 & Cephixime & Cephalosporin & CEP & $5 \mu \mathrm{g}$ & Hi Media \\
\hline 06 & Gentamycin & Aminoglycoside & GEN & $10 \mu \mathrm{g}$ & Hi Media \\
\hline 07 & Erytromycin & Macrolide & $\mathrm{E}$ & $15 \mu \mathrm{g}$ & Hi Media \\
\hline 08 & Vancomycin & Lincosamides & VA & $30 \mu \mathrm{g}$ & Hi Media \\
\hline 09 & Kanamycin & Aminoglycoside & KAN & $30 \mu \mathrm{g}$ & Hi Media \\
\hline 10 & Azithromycin & Macrolide & $\mathrm{AZ}$ & $5 \mu \mathrm{g}$ & Hi Media \\
\hline 11 & Nalidixic acid & $\begin{array}{l}\text { Quinolones and } \\
\text { Fluoroquinolones }\end{array}$ & NALI & $30 \mu \mathrm{g}$ & Hi Media \\
\hline 12 & Chloramphenicol & Chloramphenicol & $\mathrm{C}$ & $30 \mu \mathrm{g}$ & Hi Media \\
\hline 13 & Cephotaxime & Cephalosporin & $\mathrm{CE}$ & $30 \mu \mathrm{g}$ & Hi Media \\
\hline
\end{tabular}


was prepared by adding $1 \mathrm{ml}$ of concentrated sulfuric acid to $99 \mathrm{ml}$ of water and mixed well. A $1.175 \% \mathrm{~W} / \mathrm{V}$ solution of barium chloride was prepared by dissolving $2.35 \mathrm{~g}$ of dehydrated barium chloride $\left(\mathrm{BaCl}_{2} \cdot \mathrm{H}_{2} \mathrm{O}\right)$ in 200 $\mathrm{ml}$ of distilled water. To make the turbidity standard, $0.5 \mathrm{ml}$ of the barium chloride solution was added to $1 \% 99.5$ $\mathrm{ml}$ sulfuric acid solution and mixed well. A small volume of those turbid solutions was transferred to a screwcapped tube of the same type as used for preparing the control inocula and stored in the dark at room temperature. Test tubes containing different concentration $(2,4,8,16$ and $32 \mu \mathrm{g} / \mathrm{ml})$ of antimicrobial agent and the control test tube without antimicrobial agent were spot inoculated with a $2 \mu \mathrm{l}$ suspension with micropipette. Inoculation was done from the plate containing lowest concentration of antimicrobial agent and the control plate was inoculated finally. Inoculated test tube was incubated at $35^{\circ} \mathrm{C}$ for overnight. The MIC is the lowest concentration of the agent that completely inhibits visible growth was judged by the naked eye. The concentration in which the test tube showed no growth was considered as the MIC of the specific antimicrobial agent.

\subsection{Determination of MBC}

The minimum bactericidal concentration (MBC) is the concentration of antibiotic that kills at least $99.9 \%$ of a standardized bacterial inoculum. From each of the test tube where MIC was performed, $1 \mathrm{ml}$ was taken in a sterile petriplate and dispensed $15 \mathrm{ml}$ of melted pre sterilized Mueller-hinton media, mixed by rotating both clockwise and anticlockwise. Allowed to solidify, incubated at $35^{\circ} \mathrm{C}$ for overnight. The lowest concentration at which no growth occurs that was considered as the MBC of the specific antimicrobial agent.

\section{Results}

Clinical samples were collected from a total 105 indoor and outdoor patients attending at the Chittagong Maa-OShishu General Hospital, (CMOSH) during the study period. Among these samples only $11.43 \%$ of the total samples comprises Pseudomonas aeruginosa.

\subsection{Prevalence of Pseudomonas aeruginosa}

Pseudomonas aeruginosawere isolated 41.67\% (5) from blood samples, 25\% (3) from urine samples and 33.33\% (4) from pus samples. This data also represent that most of the Pseudomonas aeruginosawere isolated from blood samples (Figure 1).

\subsection{Antibiotic Resistancy Pattern of Pseudomonas aeruginosa}

In present study the isolates of Pseudomonas aeruginosa showed a pattern of resistance Ampicillin 92\%, Amoxicillin 92\%, Tetracycline 84\%, Ciprofloxacin 24\%, Cephixime 92\%, Gentamycin 55\%, Erythromycin 92\%, Vancomycin 100\%, Kanamycin 62\%, Azithromycin 32\%, Nalidixic acid 62\%, Chloramphenicol 24\% and Ce-

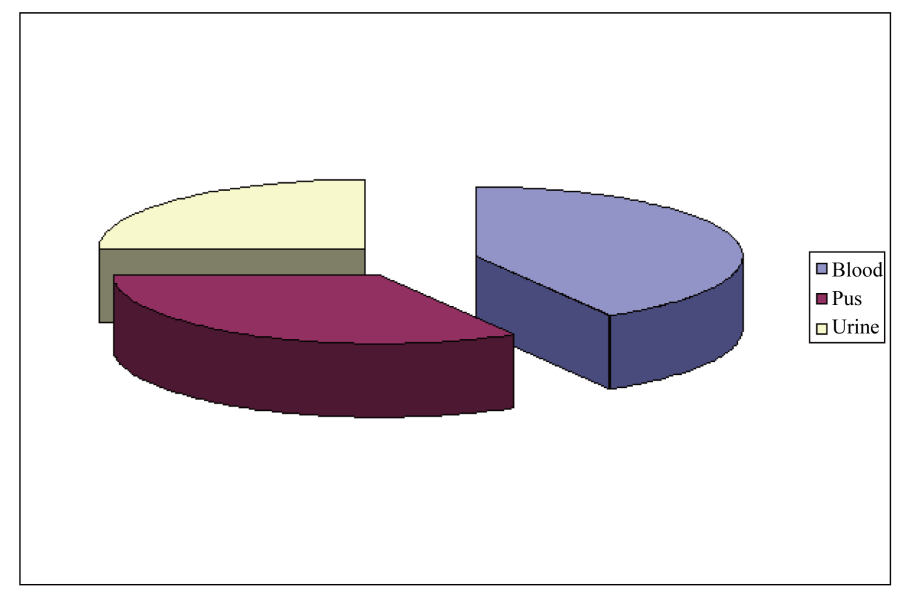

Figure 1. Percentages of specimen from where Pseudomonas aeruginosa were isolated. 
photaxime 24\%. Among these antibiotic Vancomycin, Erythromycin, Cephixime, Ampicillin, Amoxicillin and Tetracycline were highly resistant. On the other hand Ciprofloxacin, Chloramphenicol and Cephotaxime showed more sensitivity against these Pseudomonas aeruginosa isolates (Figure 2).

\subsection{The MIC and MBC Pattern of Pseudomonas aeruginosa}

On the basis of minimum inhibitory concentrations, 50\% Pseudomonas aeruginosa isolates were highly susceptible (MIC $<16 \mu \mathrm{g} / \mathrm{mL}$ ) for Ciprofloxacin, $42 \%$ isolates were susceptible (MIC $<32 \mu \mathrm{g} / \mathrm{mL}$ ) for Gentamycin and only 9\% isolates were susceptible (MIC $<32 \mu \mathrm{g} / \mathrm{mL}$ ) for Erythromycin. The range of MIC for Ciprofloxacin, Gentamycin and Erythromycin lied within 2 to $>64 \mu \mathrm{g} / \mathrm{mL}$ (Figure 3). On the other hand, the MIC for kanamycin of Pseudomonas aeruginosa isolates were 34\% (MIC $<32 \mu \mathrm{g} / \mathrm{mL}$ ), $50 \%$ isolates were susceptible (MIC $<8$

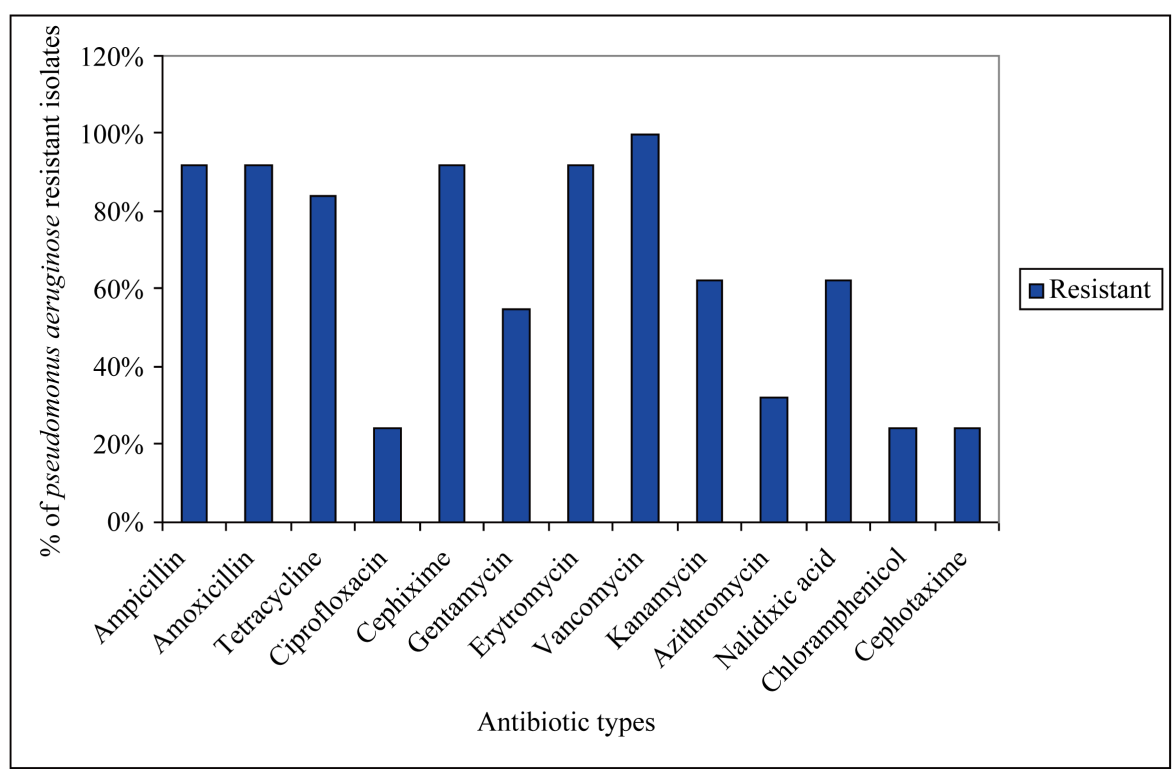

Figure 2. Percentages of Pseudomonas aeruginosa resistant isolates against thirteen antibiotic.

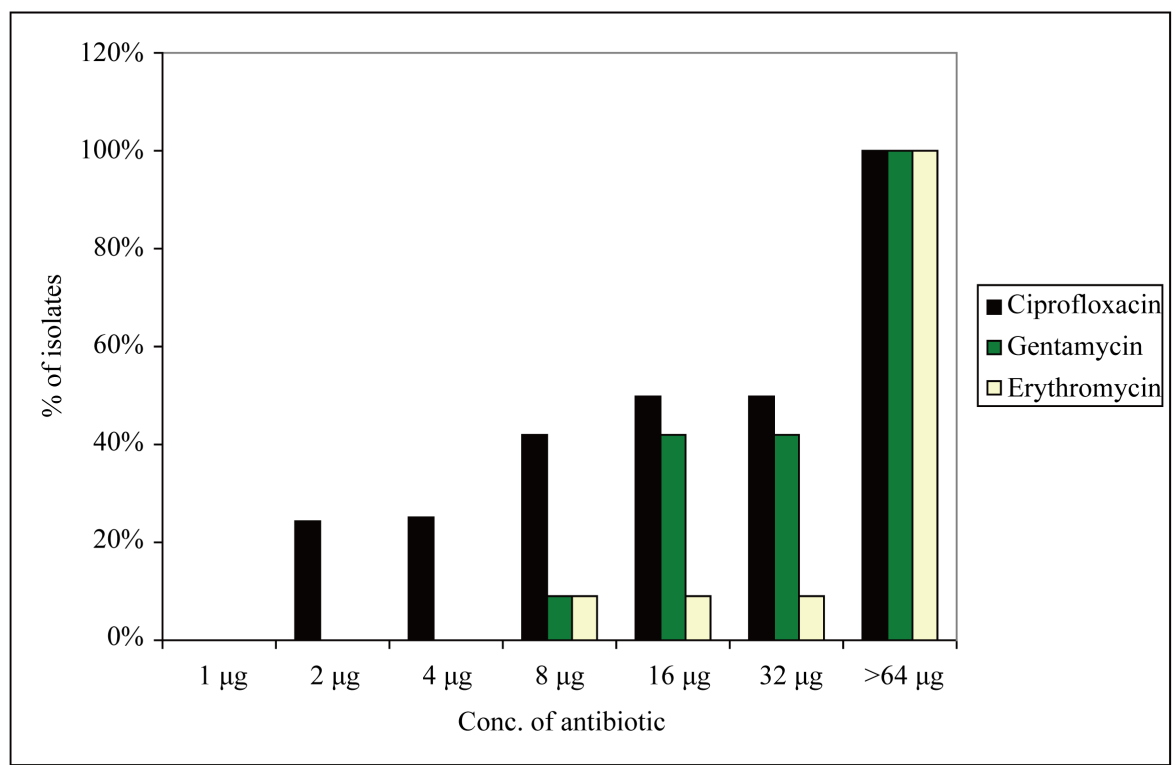

Figure 3. MIC shown by Pseudomonas aeruginosa against three antibiotics (Ciprofloxacin, Gentamycin, Erythromycin). 
$\mu \mathrm{g} / \mathrm{mL}$ ) for Azithromycin and only $42 \%$ isolates were susceptible (MIC $<16 \mu \mathrm{g} / \mathrm{mL}$ ) for Chloramphenicol (Figure 4).

A similar pattern of MBC was showed in Figure 5, Figure 6. Most of the isolates were killed at $>64 \mu \mathrm{g} / \mathrm{mL}$. Ciprofloxacin and other two brands had MBC ranging between $2 \mu \mathrm{g} / \mathrm{mL}$ to $>64 \mu \mathrm{g} / \mathrm{mL}$ (Figure 5, Figure 6).

\section{Discussion}

Pseudomonas aeruginosa is recognized as one of the most important bacterial pathogens contributing towards hospital infection worldwide. This bacteriacauses localized infection spreading into the blood stream [12]. Nosocomial infection caused by Pseudomonas aeruginosa is one of the major concerns throughout the globe since it

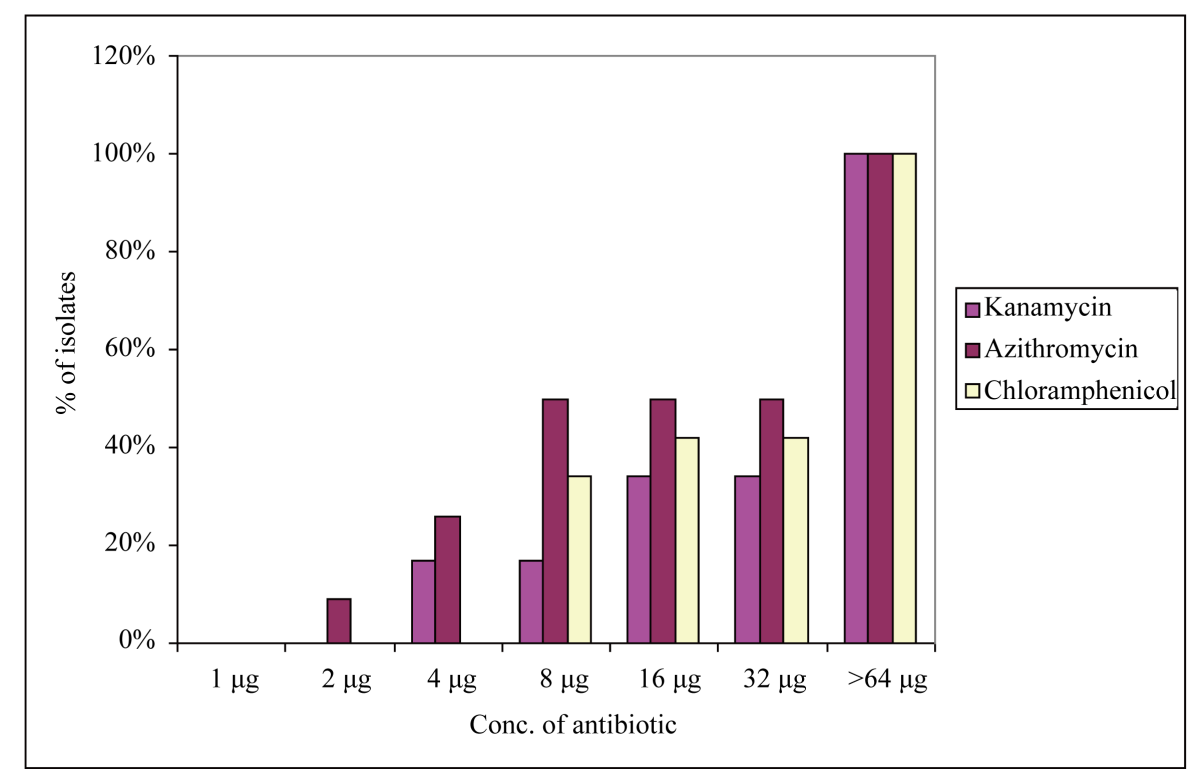

Figure 4. MIC shown by Pseudomonas aeruginosa against three antibiotics (Kanamycin, Azithromycin, Chloramphenicol).

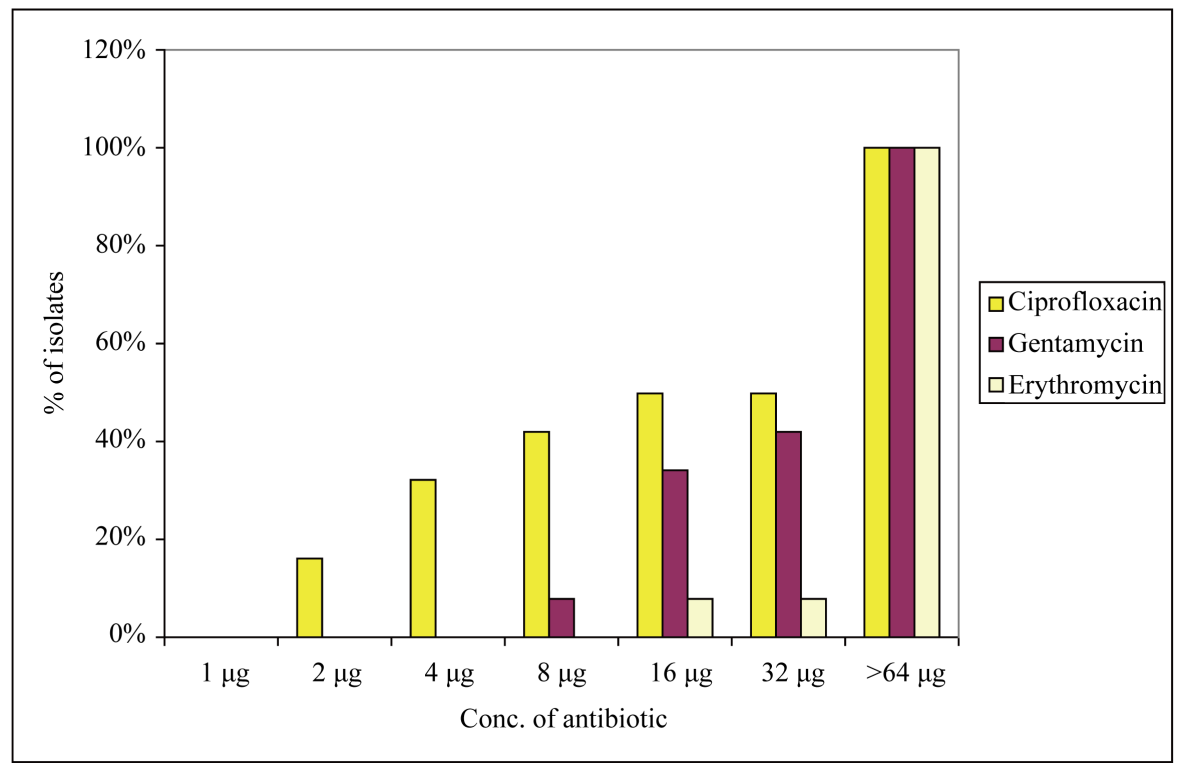

Figure 5. MBC shown by Pseudomonas aeruginosa against three antibiotics (Ciprofloxacin, Gentamycin, Erythromycin). 


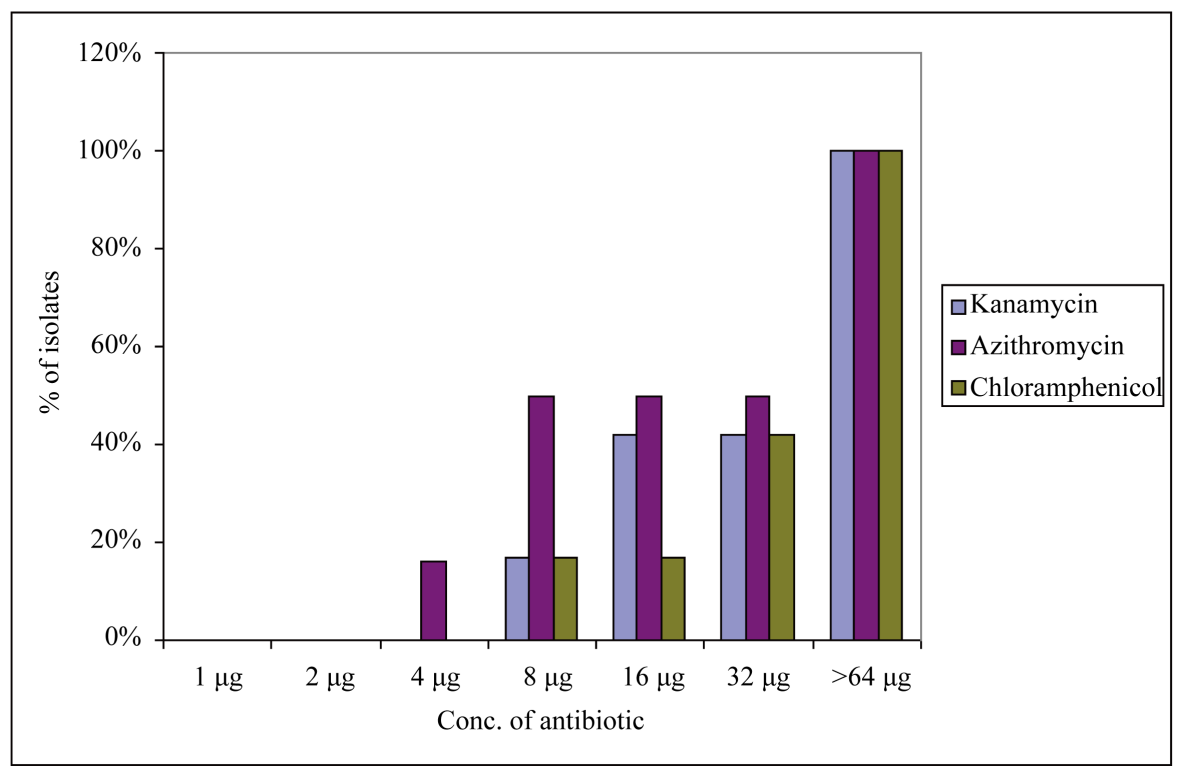

Figure 6. MBC shown by Pseudomonas aeruginosa against three antibiotics (Kanamycin, Azithromycin, Chloramphenicol).

is one of the major health hazards. Antimicrobial resistance of hospital acquired Pseudomonas aeruginosa isolates is an added disadvantage, which also confers an additional period of hospital stay and hence increasing the cost of patient care.

The present dissertation was carried out on clinical isolates from pathology laboratory of Chittagong Maa-OShishu General Hospital from in and out patients departments, to determine the prevalence of clinically significant Pseudomonas aeruginosa. About 16 isolates of Pseudomonas aeruginosa were isolated from urinary tract infected patient and also tested the antibiotic susceptibility of these isolates. A similar study was carried out by M. I. Abou-Dobara [13] and Ashok Kumar [14]. In the present research it was found that the increase in antibiotic resistance in Pseudomonas aeruginosa isolates is alarming and many antibiotics which were once thought to be the first choice in the treatment against pathogenic organisms. Although Ciprofloxacin are now largely being prescribed for the treatment of Pseudomonas aeruginosa for their less side effects \& pharmacology, it has been seen that these drugs are now becoming more insensitive. Penicillin showed the highest percentage of antibiotic resistance. It needs to mention that the semi synthetic Penicillin drug, Ampicillin was used rather than the natural Penicillin throughout this dissertation. So it is evident that the partial synthetic nature of Penicillin is not furnishing this group of drugs with enough effectiveness against these nosocomial pathogens. It has also been seen from the present study that the Ciprofloxacin and Azithromycin has been the effective drug in the treatment of Pseudomonas aeruginosa infections. Minimum Inhibitory Concentration (MIC) values of Pseudomonas aeruginosa were determined against the six antibiotics. In this study it was also found that maximum antibiotic shows MIC value greater than $64 \mu \mathrm{g} / \mathrm{mL}$ which is way too high. Here the most effective drug Ciprofloxacin and Azithromycin, the MIC value of these drugs $2-16 \mu \mathrm{g} / \mathrm{mL}$ (about 50\%) and which represent that these 3rd generation drugs become almost resistant to these two common human pathogens. MBC is not always same as the value of MIC. Some time MBC is two fold or three hold higher [15]. The MBC value of Ciprofloxacin and Azithromycin lies $2-32 \mu \mathrm{g} / \mathrm{mL}$ (about 50\%) which strongly support that to kill the bacteria more amount of antibiotic were required. Inappropriate use increases the risk for selection and dissemination of antibiotics-resistant bacteria, which are placed at a competitive advantage. Therefore, the drugs, which are commonly used, lead to development of bacterial resistance in developing countries, which are generally inexpensive, and popular broad spectrum agent [16]-[18]. The emergence and spread of bacterial resistance due to use of antibiotic, is a complex phenomenon. Antibiotic use in clinical practice alone can not explain the high frequency of resistant microorganism [19] [20]. Moreover, the excessive misuse is practically responsible for development of increased rate of antibiotic resistance, globally. Another factor responsible for development of resistance in bacteria could be due to non-access of health workers to health information [21]. In most of the developing countries including Bangladesh, antibiotics 
can be purchased without prescription and "available on prescription" is just confined on the tag of the medicine only. In Bangladesh there is virtually no difference between a non-prescription available over the counter drug like paracetamol and a prescription antibiotic. Drug labels and packages inserts often fail to provide precise information [22] and in industrialized countries patients often pressurize physicians to prescribe antibiotics [23]. Another bemoaning finding of this study is that in Bangladesh medical practitioners prescribe drugs with proprietary names rather than the generic name. For an instance, if a patient is suffering from typhoid will most probably be prescribed Fluoroquinolones like Ciprofloxacin or Ofloxacin but in practice, it is evident that practitioners are rather prescribing a proprietary drug of their choice and there is allegation against many physicians from various sources that they are receiving a good amount of incentive from the pharmaceutical companies for prescribing their drugs. It was also noted that all patients were habitual to self-medication and these patients also visited private practitioners during different periods of time in present study most of the isolates were resistant to Penicillin (Amphicillin and amoxicillin) (over 80\%) this could be due to the excessive use of these drug.

\section{Conclusion}

This study documents the importance of Pseudomonas aeruginosa as an important Gram-negative pathogen among Bangladeshi populations and highlights the facts that there is an alarming development of multidrug resistance among clinical isolates. This study also alarms us to stop the misuse of antibiotic which day by day increases the multi-drug resistance problem.

\section{References}

[1] Buchanon, U.R.E. and Gibbons, N.E. (1974) Bergey’s Manual of Determinative Bacteriology. 8th Edition, The Williams and Wilkins Company, Baltomore.

[2] Brooks, G.F., Butel, J.S. and Moore, S.A. (2004) Jawetz, Melnick and Adelberg's Medical Microbiology. 23rd Edition, McGraw-Hill/Appleton and Lange, New York.

[3] Todar, K. (2004) Todar's Online Textbook of Bacteriology: Pseudomonas aeruginosa. Department of Bacteriology, University of Wisconsin, Madison.

[4] Rayner, C.F.J., Cole, P.J. and Wilson, R. (1994) The Management of Chronic Bronchial Sepsis Due to Bronchiectasis. Clinical Pulmonary Medicine, 1, 348-355. http://dx.doi.org/10.1097/00045413-199411000-00002

[5] Seol, B., Naglic, T., Madic, J. and Bedekovic, M. (2002) In Vitro Antimicrobial Susceptibility of 183 Pseudomonas aeruginosa Strains Isolated from Dogs to Selected Antipseudomonal Agents. Journal of Veterinary Medicine, 49, 188192. http://dx.doi.org/10.1046/j.1439-0450.2002.00548.x

[6] Li, X.Z., Livermore, D.M. and Nikaido, H. (1994) Role of Efflux Pump(s) in Intrinsic of Pseudomonas aeruginosa: Resistance to Tetracycline, Chloramphenicol and Norfloxacin. Antimicrob. Antimicrobial Agents and Chemotherapy, 38, 1732-1741. http://dx.doi.org/10.1128/AAC.38.8.1732

[7] Schaber, J.A., Carty, N.L., MacDonald, N.A., Graham, E.D., Cheluvappa, R., Griswold, J.A. and Hamood, A.N. (2004) Analysis of Quorum Sensing-Dificient Clinical Isolates of Pseudomonas aeruginosa. Journal of Medical Microbiology, 53, 841-853. http://dx.doi.org/10.1099/jmm.0.45617-0

[8] Wilson, R. and Dowling, R. (1998) Pseudomonas aeruginosa and Other Related Species. Thorax, 53, 231-219. http://dx.doi.org/10.1136/thx.53.3.213

[9] Benson, H.J. (1998) Antimicrobial Sensitivity Testing: the Kirby-Bauer Method. In: Benson, H.J., Ed., Microbiological Applications: Laboratory Manual in General Microbiology, 7th Edition, McGraw Hill, Boston, 139-141.

[10] (2003) National Committee for Clinical Laboratory Standards, Methods for Dilution Antimicrobial Susceptibility Tests for Bacteria That Grow Aerobically; Approved Standard M7-A6, National Committee for Clinical Laboratory Standards, Wayne.

[11] National Committee for Clinical Laboratory Standards, Performance Standards for Antimicrobial Susceptibility Testing (2003) M100-S13. National Committee for Clinical Laboratory Standards, Wayne.

[12] Brodie, S.B., Sands, K.E., Gray, J.E., Parker, R.A., Goldmann, D.A., Davis, R.B. and Richardson, D.K. (2000) Occurrence of Nosocomial Bloodstream Infections in Six Neonatal Intensive Care Units. Pediatric Infectious Disease Journal, 19, 56-65. http://dx.doi.org/10.1097/00006454-200001000-00012

[13] Abou-dobara, M.I., Deyab, M.A., Elsawy, E.M. and Mohamed, H.H. (2010) Antimicrobial Susceptibility Typing and Genotyping Pattern of Escherichia coli, Klebsiella pneumoniae and Pseudomonas aeruginosa Isolated from Urinary Tract Infected Patients. Polish Journal of Microbiology, 59, 207-212.

[14] Kumar, A. and Talwar, A. (2010) Study on the Presence of K. pneumonia and K. oxytocain Raw Milk and Antibiotic 
Susceptibility Testing. Human Ecology, 32, 207-208.

[15] Visser, M.R., Arska, M.R., Beumer, H., Hoepelman, I.M. and Verhoef, J. (1991) Comparative in Vitro Antibacterial Activity of Sparfloxacin (AT-4140; RP 64206), a New Quinolone. Antimicrobial Agents and Chemotherapy, 35, 858868. http://dx.doi.org/10.1128/AAC.35.5.858

[16] Calva, J.J., Sifuentes-Osornio, J. and Ceron, C. (1996) Antimicrobial Resistance in Fecal Flora: Longitudinal Community-Based Surveillance of Children from Urban Mexico. Antimicrobial Agents and Chemotherapy, 40, 1699-1702.

[17] Sack, R.B., Rahman, M., Yunus, M. and Khan, E.H. (1997) Antimicrobial Resistance in Organisms Causing Diarrheal Disease. Clinical Infectious Diseases, 24, S102-S105. http://dx.doi.org/10.1093/clinids/24.Supplement_1.S102

[18] Hoge, C.W., Gambel, J.M., Srijan, A., Pitarangsi, C. and Echeverria, P. (1998) Trends in Antibiotic Resistance among Diarrheal Pathogens Isolated in Thailand over 15 Years. Clinical Infectious Diseases, 26, 341-345. http://dx.doi.org/10.1086/516303

[19] Col, N.F. and O’Connor, R.W. (1987) Estimating Worldwide Current Antibiotic Usage: Report of Task Force 1. Clinical Infectious Diseases, 9, S232-S243. http://dx.doi.org/10.1093/clinids/9.Supplement_3.S232

[20] Kunin, C.M. (1993) Resistance to Antimicrobial Drugs-A Worldwide Calamity. Annals of Internal Medicine, 118, 557-561. http://dx.doi.org/10.7326/0003-4819-118-7-199304010-00011

[21] Carmeli, Y., Troillet, N., Eliopoulos, G.M. and Samore, M.H. (1999) Emergence of Antibiotic-Resistant Pseudomonas aeruginosa: Comparison of Risks Associated with Different Antipseudomonal Agents. Antimicrobial Agents and Chemotherapy, 43, 1379-1382.

[22] Lee, P.R., Lurie, P., Silverman, M.M. and Lydecker, M. (1991) Drug Promotion and Labeling in Developing Countries: An Update. Journal of Clinical Epidemiology, 45, 49-55. http://dx.doi.org/10.1016/0895-4356(91)90113-N

[23] Paredes, P., Pena, M., Guerra, E.F., Diaz, J. and Trostle, J. (1996) Factors Influencing Physicians’ Prescribing Behavior Ion the Treatment of Childhood Diarrhea: Knowledge May Not Be the Clue. Social Science \& Medicine, 42, 1141-1153. http://dx.doi.org/10.1016/0277-9536(95)00387-8 